\title{
Automatic Facsimile of Chinese Calligraphic Writings
}

\author{
Songhua $\mathrm{Xu}^{1,2,3 \dagger} \quad$ Hao Jiang $^{3} \quad$ Tao Jin $^{3} \quad$ Francis C.M. Lau ${ }^{3} \quad$ Yunhe Pan ${ }^{1}$ \\ ${ }^{1}$ College of Computer Science and Technology, Zhejiang University, Hangzhou, Zhejiang, P.R. China, 310027 \\ ${ }^{2}$ Department of Computer Science, Yale University, New Haven, Connecticut, USA, 06520 \\ ${ }^{3}$ Department of Computer Science, The University of Hong Kong, Hong Kong, P.R.China
}

\begin{abstract}
To imitate personal handwritings is non-trivial. In this paper, we attempt to address the challenging problem of automatic handwriting facsimile. We focus on Chinese calligraphic writings due to their rich variation in style, high artistic values and also the fact that they are among the most difficult candidates for the problem. We first analyze the structures and shapes of the constituent components, i.e., strokes and radicals, of characters in sample calligraphic writings by the same writer. To generate calligraphic writing in the style of the writer, we facsimile the individual character elements as well as the layout relationships used to compose the character, both in the writer's personal writing style. To test our algorithm, we compare our facsimileing results of Chinese calligraphic writings with the original writings. Our results are found to be acceptable for most cases, some of which are difficult to differentiate from the real ones. More results and supplementary materials are provided in our project website at http://www.cs.hku.hk/ songhua/facsimile/.
\end{abstract}

Categories and Subject Descriptors (according to ACM CCS): I.2.1 [Artificial Intelligence]: Applications and Expert Systems; I.3.3 [Computer Graphics]: Picture/Image Generation; J.5.C [Arts and Humanities]: Fine arts.

\section{Introduction and Related Work}

Chinese calligraphy is one of the most important art forms of Chinese traditional culture, as well as one of the most complicated hand-written character systems currently in use in the world. To produce facsimiles (i.e., exact copies) of Chinese calligraphic writings is an intellectual challenge, even for human beings. In this paper, we explore the possibility to achieve the challenging goal of Chinese calligraphy facsimile by the computer, i.e., to generate Chinese calligraphic characters in the style of a particular human writer. Tackling this problem has a number of applications in the practical world. For example, with a good algorithm capable of facsimileing handwriting, the computer can generate an whole document in handwritten style as if it were manually written character by character by the human author. Documents in handwriting style produced this way are more "personal" and can draw the reader closer to the author than "typed" documents.

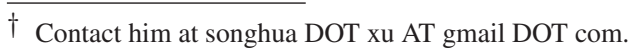

A body of work exists for solving the computational problems arising from processing calligraphic characters. Adachi and Ishii [MAI93] applied fuzzy theory to teaching computers to learn handwriting. Yamasaki and Hattori [YH96] tackled the Japanese calligraphy generation problem through treating calligraphic characters as being composed of fundamental brush strokes in a hierarchic manner, which provides a good reference for calligraphy generation in our work. Gader et al. [GKK ${ }^{*}$ 97] adopted fuzzy logic for recognizing calligraphic letters. Mano et al. [MHN*99] applied interpolation to fuzzy spline curves to generate Japanese calligraphy. Wang and Lee [WL01] studied the problem of denoising and binarization of ancient calligraphic writing images through sophisticated image processing procedures. Yu and Peng [YP05] applied image synthesis algorithms to capture the aesthetic appearance of cursive Chinese calligraphy. Wong et al. [WLI05] created Chinese calligraphic writings interactively via a geometric modeling approach. Okabe et al. [OSN05] proposed a new rendering method for generating line renditions in paintbrush styles using Hidden Markov Models. Also related is Freeman et al. [FTP03]'s 
work on style facsimile for cartoon drawings, which can capture the relationship between different artists' styles in line art drawings through a nearest-neighbour-based local learning approach. Their work inspires us directly for our character element facsimile process. More recently, Wang et al. [WWPF08] introduced a style preserving Chinese character synthesis method based on hierarchical representation of characters. Their synthesis method is based solely on reusing existent writing examples previously created by a person; in contrast, our method, in addition to reusing existent writing examples, can generate calligraphic characters not previously written by the person. Obviously, their reuse-based calligraphy synthesis is a degenerate case of our hybrid reuse-and-generation-based calligraphic writing facsimile algorithm. In our prior work, we introduced a constraint-based spatial reasoning method for the generation of novel calligraphic writings based on a few parameterized calligraphic samples [XLP03, XLCP04, XLCP05]. More recently, we introduced a neural-network-based approach to evaluate the aesthetics of Chinese calligraphy for providing feedback to guide the automatic Chinese calligraphy generation process [XJLP07]. These previous projects aimed at generating good-looking Chinese calligraphy, which is different from facsimileing a person's handwriting. Our research here is to capture and then mimic the calligraphic writing style of a particular human writer through a learningbased approach.

The rest of our paper is organized as follows. We first discuss how to decompose existent calligraphic writings and store in a personal handwriting database in Sec. 2. Since a Chinese character is an assemblage of strokes or radicals following a certain spatial layout, we discuss how to facsimile strokes and radicals in Sec. 3, and then explain how to facsimile the layout in order to compose a character in Sec. 4. We discuss how to generate an optimal facsimile result when multiple candidate results are available in Sec. 5 . We report experimental results in Sec. 6 and conclude the paper in Sec. 7.

\section{Personal Handwriting Database}

All the available calligraphic writings by the same person will first be decomposed into a hierarchy of radicals which then form the basic strokes; the results are parametrically stored in a "Personal Handwriting DataBase" (PH-DB). The decomposition process is realized through applying the stroke decomposition algorithm proposed in [XJLP07], which we consider to be one of the most suitable algorithms for extracting a hierarchical and parametric representation of Chinese calligraphic characters. The result of the parameterization is that each character element will be vectorized as a triplet $\left(\mathbf{C}_{x}, \mathbf{C}_{y}, \mathbf{C}_{w}\right)$ of which each element is a onedimensional curve. The curves of $\mathbf{C}_{x}$ and $\mathbf{C}_{y}$ give the trajectory of a stroke and the curve $\mathbf{C}_{w}$ records the width of the stroke. Two example character decomposition results are



(a)

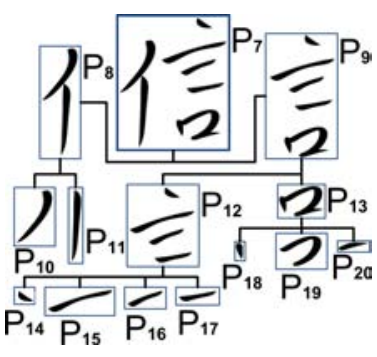

(b)
Figure 1: Two example character decomposition results.

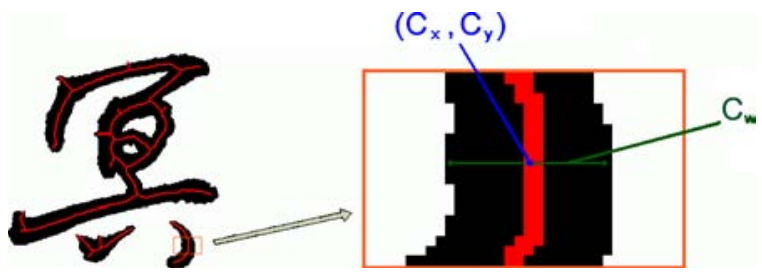

Figure 2: The curve triplet $\left(\boldsymbol{C}_{x}, \boldsymbol{C}_{y}, \boldsymbol{C}_{w}\right)$ for parametrically representing a stroke.

shown in Figure 1. Figure 2 shows how we use the curve triplet to represent a stroke decomposed from a character. To be able to apply algebraic operations including multiplication and addition to these stroke representing curves to create a new stroke, which is discussed in Sec. 3, we represent these curves discretely in the form of matrices whose dimensionalities are normalized. With such a matrix form discrete curve representation, ordinary matrix multiplication and addition operations can be applied. Some detailed discussions of matrix operations over our discrete stroke representing curves can be found in [XLCP05].

In the PH-DB, strokes and radicals decomposed from the image of a written character are called the "character's constructive elements", which we abbreviate as "character elements", or simply "elements" when there is no ambiguity. Each character element $\xi$ has a type property $\tau(\xi)$, representing the category of stroke/radical that $\xi$ belongs to according to the conventional Chinese character formation method. For example, in Figure 1, $P_{1}$ and $P_{8}$ both belong to the category of "single person's radicals", thus $\tau\left(P_{1}\right)=\tau\left(P_{8}\right)$. Similarly, $\tau\left(P_{4}\right)=\tau\left(P_{11}\right)=\tau\left(P_{18}\right)$ as they all belong to the category of "vertical strokes", and $\tau\left(P_{5}\right)=\tau\left(P_{6}\right)=\tau\left(P_{15}\right)=$ $\tau\left(P_{16}\right)=\tau\left(P_{17}\right)=\tau\left(P_{20}\right)$ because they all belong to the category of "horizontal strokes". Such a categorization is important for our algorithm as elements having the same type property will be referred to when facsimileing new elements in the category. In our prototype system implementation, we support five basic stroke types: "point stroke", "left-slanting stroke", "right-slanting stroke", "vertical stroke" and "horizontal stroke", as well as fifty most frequently used radicals in Chinese character formation. 


\section{Facsimileing Individual Character Elements}

Two potential approaches exist for facsimileing individual character elements-one is through reusing a similar element written by the same writer previously, and the other is through generating a facsimile of the element in question according to other character elements previously written by the writer. Both approaches have their pros and cons. The first, reuse-based approach can efficiently produce a correct facsimile if the element indeed has been previously written by the writer. But if the user has never written the element or a similar one before, the approach cannot create a facsimile and thus would fail. The second approach works more generically. It might not however achieve the same degree of correctness as the reuse-based approach. We therefore introduce a hybrid approach combining the two. In the following, we first discuss the reuse-based and generation-based facsimile methods in Sec. 3.1 and Sec. 3.2 respectively. After that, we introduce our hybrid method for character element facsimile in Sec. 3.3.

\subsection{Reuse-based character element facsimile}

We rely on the character context to evaluate the fitness of reusing one character element previously written by a person for facsimileing a written character element $\xi$. Recall in Sec. 2, we acquire all the available sample character elements written by a person through character decomposition. For a character that has the character element $\xi$ in the character's composition, we find $\xi$ 's parent element in the character's hierarchical construction tree. For example, in Figure $1, P_{1}$ 's parent element is $P_{0}$ and $P_{16}$ 's parent element is $P_{12}$. We can also find $\xi$ 's ancestor elements via the construction tree. Here we denote $\xi$ 's $i$-th ancestor element as $A n c_{i}(\xi)$. We also represent the maximum level of $\xi$ 's ancestors as $\gamma(\xi)$. Assuming $\xi$ is at the $x$-th level in the character construction tree, then $\gamma(\xi)=x-1$. For $i>\gamma(\xi), A n c_{i}(\xi)$ does not exist because the root of the tree (i.e., the whole character) is $A n c_{\gamma(\xi)}(\xi)$. For this situation, we specifically define $A n c_{i}(\xi)=N U L L$ for $i>\gamma(\xi)$. Revising the examples shown in Figure $1, A n c_{0}\left(P_{3}\right)=P_{3}$, $A n c_{1}\left(P_{3}\right)=P_{1}, A n c_{2}\left(P_{3}\right)=P_{0}, A n c_{3}\left(P_{3}\right)=N U L L, \gamma\left(P_{3}\right)=$ 2 and $A n c_{0}\left(P_{15}\right)=P_{15}, A n c_{1}\left(P_{15}\right)=P_{12}, A n c_{2}\left(P_{15}\right)=P_{9}$, $\operatorname{Anc}_{3}\left(P_{15}\right)=P_{7}, \operatorname{Anc}_{4}\left(P_{15}\right)=N U L L, \gamma\left(P_{15}\right)=3$. Given these notations, we can now define the character context similarity between two character elements $\xi$ and $\xi^{\prime}$ as:

$$
\Delta\left(\xi, \xi^{\prime}\right) \triangleq \sum_{j=0}^{\max \left\{\gamma(\xi), \gamma\left(\xi^{\prime}\right)\right\}}\left(z_{j} \cdot \delta\left(A n c_{j}(\xi), A n c_{j}\left(\xi^{\prime}\right)\right)\right)
$$

where

$$
\delta(a, b)= \begin{cases}1 & \text { if } \tau(a)=\tau(b), \text { and } a, b \neq N U L L ; \\ 0 & \text { otherwise. }\end{cases}
$$

Here $z_{j}$ 's are the fall-off coefficients, defined as $z_{j}=$ $2 /\left(3^{j+1}\right)$. The purpose of introducing these fall-off coefficients is to emphasize more the local context in the charac- ter construction hierarchy than the remote context. Such a similarity measurement reflects the character context around the character element to be facsimiled, which is used as the most important information in determining whether a character element shall be reused for the facsimileing or not. A larger value of $\Delta\left(\xi, \xi^{\prime}\right)$ suggests more suitability to use $\xi^{\prime}$ as a facsimile for $\xi$. The value range for $\Delta\left(\xi, \xi^{\prime}\right)$ is between 0 and 1.

Given this content similarity metric, the search for a most suitable element to be used as the facsimile over $\xi$ in the writer's style is reduced to searching among all the character elements stored in the person's PH-DB which can maximize the character context similarity with $\xi$, i.e., to find a $\xi_{i}$ so that $\xi_{i}=\arg \max _{\xi_{j}} \Delta\left(\xi, \xi_{j}\right)$. More specifically, we look for ten $\xi_{i}$ 's that yield the highest similarity scores with $\xi$ to form a candidate set of facsimile results $\Omega(\xi)$. We then randomly select the character element $\xi_{i}$ in $\Omega(\xi)$ as the facsimile result for $\xi$ with a probability of $p_{i}$. The probabilities $p_{i}$ 's controlling the random selection process are defined in (2), which implement a random selection mechanism with the property that the more similar $\xi_{i}$ is to $\xi$ by character context, the more likely $\xi_{i}$ will be selected as the facsimile result over $\xi$.

$$
p_{i} \triangleq \frac{\Delta\left(\xi_{i}, \xi\right)}{\sum_{\xi_{j} \in \Omega(\xi)} \Delta\left(\xi_{j}, \xi\right)} .
$$

\subsection{Generation-based character element facsimile}

We denote the shape of the character element $\xi$ in the personal handwriting style of the writer $X$ as $\xi(X)$. To generate it, we look for the element's handwritings by other writers in $\mathrm{PH}-\mathrm{DB}$, which are denoted as $\operatorname{Ref}(\xi) \triangleq$ $\left\{\xi\left(W_{1}\right), \xi\left(W_{2}\right), \cdots, \xi\left(W_{n w}\right)\right\}$. Note that standard fonts from a commercial font system are also stored in our PH-DB and thus $\operatorname{Ref}(\xi)$ would never be empty since the font system comprehensively covers all the character elements. Each standard font is treated as one separate writer's style. In our experiment, we adopt ten popular Chinese fonts, most of which have come with the Microsoft Word software. In a nutshell, the way we generate $\xi(X)$ is to find an optimal linear combination of these samples to approximate $\xi(X)$, i.e.:

$$
\xi(X) \approx \sum_{i=1}^{n w} \lambda_{i} \xi\left(W_{i}\right)
$$

in which $\lambda_{i}$ 's are the combination weights satisfying $\sum_{i=1}^{n w} \lambda_{i}=1$. To determine the most suitable weight configuration, we first search for ten character elements $\xi_{j}(j=$ $1, \cdots, 10)$ which have already been written by $X$, as well as by the writers $W_{1}, \cdots, W_{n w}$. If there are more than ten such elements, we select ten of them which achieve the highest content similarity scores with $\xi$ according to (1). Given a certain assignment over $\lambda_{i}$ 's, we can approximate $\xi_{j}(X)$ based on the linear combination of $\xi_{j}\left(W_{1}\right), \cdots, \xi_{j}\left(W_{n w}\right)$ through a form similar to (3), i.e., $\xi_{j}(X) \approx \sum_{i=1}^{n w} \lambda_{i} \xi_{j}\left(W_{i}\right)$. We derive the approximation error as $e_{j} \triangleq\left|\xi_{j}(X)-\sum_{i=1}^{n w} \lambda_{i} \xi_{j}\left(W_{i}\right)\right|$. 
This error is measured as the number of pixels that are different in the corresponding positions in the binary images of the element $\xi_{j}(X)$ and $\sum_{i=1}^{n w} \lambda_{i} \xi_{j}\left(W_{i}\right)$. We optimally determine $\lambda_{i}$ 's in (3) for facsimileing $\xi(X)$ through finding an optimal assignment over $\lambda_{i}$ which can minimize $\sum_{j=1}^{n w}\left|e_{j} \Delta\left(\xi_{,}, \xi_{j}\right)\right|$. We use a simulated annealing process to solve the optimization problem and denote the optimal assignment so found over $\lambda_{i}$ 's as $\lambda_{1}^{\text {opt }}(\xi), \cdots, \lambda_{n w}^{\text {opt }}(\xi)$. Here the parameter " $\xi$ " in brackets means such assignment over $\lambda_{i}$ 's is optimized for facsimileing the character element $\xi$.

\subsection{A hybrid method for character element facsimile}

As mentioned earlier, if the reuse-based facsimile approach works out, the facsimile result expectedly has a higher facsimile faithfulness than what may be achieved by the generation-based approach. In situations where the reusebased approach may not work out because of the lack of sufficient reference samples, we introduce a mechanism which randomly outputs the facsimile result by the reuse-based approach with a probability of $\alpha$ and outputs the result of the generation-based approach with a probability of $1-\alpha$ where $\alpha$ is defined as $\alpha \triangleq \frac{1}{|\Omega(\xi)|} \sum_{\xi_{j} \in \Omega(\xi)} \Delta\left(\xi_{j}, \xi\right)$ where $|\Omega(\xi)|$ returns the cardinality of the set $\Omega(\xi)$. Recall at the end of Sec. 3.1 we define $\Omega(\xi)$ as the set of candidate character element facsimile results for $\xi$. It is easy to see that the higher the average character context similarity between $\xi$ and $\xi_{j}(j=1, \cdots, n)$, the more likely our algorithm will output results produced by the reuse-based facsimile approach rather than the generation-based approach.

\section{Facsimileing Character Layout}

After all the character elements needed for composing a character $\xi$ have been facsimiled through the procedure discussed in Sec. 3, we need to facsimile the layout into which these elements of $\xi$ are assembled. Similar to our method for facsimileing character elements as studied in Sec. 3, we propose also a hybrid method for facsimileing the spatial layout of a character due to the same reason-reuse-based layout facsimileing will fail if the reference samples are not plenty enough whereas generation-based facsimileing may succeed even when the system only has access to very limited samples. In situations when they both work, the quality of the results produced by reuse-based facsimile is generally better than that of the generation-based approach. Since both reuse-based and generation-based approaches need to refer to layout examples for facsimileing a character's composition layout, we first look at the way to efficiently find relevant spatial layout examples in Sec. 4.1. And then we discuss our method for facsimileing the spatial layout of a character according to the retrieved example layouts from the personal handwriting database in Sec. 4.2.

\subsection{Efficiently finding relevant character layout examples}

Here we first look at how to characterize the spatial layout of a character according to a few layout features organized as feature matrices (Sec. 4.1.1). Based on these matrices, we can categorize all the known spatial layouts stored in the personal handwriting database in advance so that our algorithm can efficiently retrieve relevant layout examples on the fly (Sec. 4.1.2). With these preprocessing work, we can efficiently find relevant layout examples (Sec. 4.1.3).

\subsubsection{Feature matrices of character layout}

To facsimile the layout of a character $\xi$, we assume the character is composed of $n$ character elements in the lower levels of the character construction hierarchy. We denote that as $S(\xi)=\widehat{\xi_{1}} \ldots \widehat{\xi_{n}}$ where $\widehat{\xi_{1}}, \cdots, \widehat{\xi_{n}}$ are the character elements at the lower levels of the character constructive tree of the character $\xi$. We call $S(\xi)$ a constructive approach to the character $\xi$. Note that for the same character, there could exist multiple possible constructive approaches because some approaches utilize radicals to compose the character, some use strokes and some use both. For a particular constructive approach $S(\xi)=\widehat{\xi_{1}} \ldots \widehat{\xi_{n}}$, we derive each character element $\hat{\xi}_{i}$ 's bounding box as $B_{i}$. We also denote $B_{i}$ 's center coordinates as $\left(x_{i}, y_{i}\right)$ and its width and height as $w_{i}$ and $h_{i}$ respectively. For every pair of elements $\widehat{\xi}_{i}$ and $\widehat{\xi}_{j}$ in $S(\xi)$, we calculate two values $f_{x}\left(\widehat{\xi}_{i}, \widehat{\xi}_{j}\right)$ and $f_{y}\left(\widehat{\xi}_{i}, \widehat{\xi}_{j}\right)$ to characterize the spatial relationship between $\widehat{\xi}_{i}$ and $\widehat{\xi}_{j}$ :

$$
\left\{\begin{array}{l}
f_{x}\left(\widehat{\xi}_{i}, \widehat{\xi}_{j}\right) \triangleq\left(x_{i}-x_{j}\right) /\left(w_{i}+w_{j}\right) \\
f_{y}\left(\widehat{\xi}_{i}, \widehat{\xi}_{j}\right) \triangleq\left(y_{i}-y_{j}\right) /\left(h_{i}+h_{j}\right)
\end{array},\right.
$$

Based on these two spatial layout features, we can further construct two matrices $\mathbf{F}_{x}$ and $\mathbf{F}_{y}$ each of dimensionality $n \times$ $n$ to characterize the spatial layout inside the character. The elements on the $i$-th row and $j$-th column of the matrices $\mathbf{F}_{x}$ and $\mathbf{F}_{y}$ are the corresponding $f\left(\widehat{\xi}_{i}, \widehat{\xi}_{j}\right)$ values, i.e.: $\mathbf{F}_{x}(i, j)=$ $f_{x}\left(\widehat{\xi}_{i}, \widehat{\xi}_{j}\right)$ and $\mathbf{F}_{y}(i, j)=f_{y}\left(\widehat{\xi}_{i}, \widehat{\xi}_{j}\right)$.

\subsubsection{Categorizing character layout examples}

In the traditional Chinese character formation method, all the Chinese characters conform to some rectangular structures. For example, the two characters shown in Figure 1 are both composed of two radicals in a horizontal manner, i.e., $P_{0}=P_{1} P_{2}$ and $P_{7}=P_{8} P_{9}$. This is conventionally called the "left-right structure" (Figure 3(b)). A constructive approach $S(\xi)=\widehat{\xi_{1}} \ldots \widehat{\xi_{n}}$ for a certain character $\xi$ written by a certain writer $X$ corresponds to a set of bounding boxes, $\left\{B_{i} \mid i=1, \cdots, n\right\}$, where $B_{i}$ is the bounding box of the character element $\widehat{\xi}_{i}$. The spatial relationships between these bounding boxes define a layout for the character in the constructive approach. To make our layout facsimile process more reliable and also more efficient to execute on the fly, 




(a)
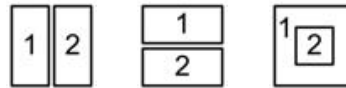

欣

隽

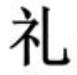

(b)

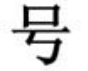

(c)
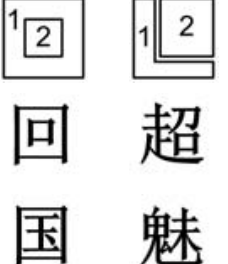

(d)
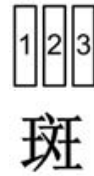

魅

(e) as $L\left(S(\xi), W_{\text {std }}\right)$. We assume even though each writer has his personal handwriting style, the variation of his personal handwriting style from the standard font style shall not affect the category to which the layout belongs. That is, if $L\left(S(\xi), W_{\text {std }}\right)$ is a "left-right" structure as shown in Figure 3, then $L(S(\xi), X)$ shall also be "left-right" structured. We feel this is a safe assumption as otherwise characters written in that person's style would not be easily recognized by other people. Under the above assumption, we first classify the layout $L\left(S(\xi), W_{\text {std }}\right)$ into one of the layout classes via the layout classification procedure discussed in Sec. 4.1.2. Without loss of generality, we assume it falls into the layout class of $T_{i}$. Among all the layout relationships in $T_{i}$, we find ten most similar layouts to $L(S(\xi), X)$. The distance metric $\Theta\left(L_{a}, L_{b}\right)$ we use to evaluate the similarity between two layouts $L_{a}$ and $L_{b}$ is very similar to the layout similarity function $\Theta\left(L(S(\xi), X), T_{i}\right)$ defined in (5) except that we compare the sets of bounding boxes corresponding to the two constructive approaches rather than a construction approach and a standard layout example. The layout thus found might be collected from characters previously written by the writer $X$ or by other writers. We denote the layout found which belongs to the first case $\quad$ as $\quad \mathbf{L}_{\text {self }} \triangleq\left\{L\left(S_{1}^{\text {self }}\left(\xi_{1}\right), X\right), \cdots, L\left(S_{s n}^{\text {self }}\left(\xi_{s n}\right), X\right)\right\}$ (assuming there are $s n$ of them) and templates found which belong to the latter case as $\mathbf{L}_{\text {other }} \triangleq$ $\left\{L\left(S_{1}^{\text {other }}\left(\xi_{1}\right), W_{1}\right), \cdots, L\left(S_{\text {on }}^{\text {other }}\left(\xi_{\text {on }}\right), W_{\text {on }}\right)\right\} \quad$ (assuming there are on of them).

\subsection{Character layout facsimileing}

\subsubsection{Reuse-based character layout facsimileing}

In this facsimile approach, we try to facsimile the layout of a character through reusing one of the character layout examples in $\mathbf{L}_{\text {self }}$. We feel this reuse-based facsimile is a safe move because the layout example to be reused is produced by the same writer. More concretely, we randomly select the layout example $L\left(S_{i}^{\text {self }}\left(\xi_{i}\right), X\right)$ from $\mathbf{L}_{\text {self }}$ with a probability $q_{i}$ when facsimileing the character layout $L(S(\xi), X)$. The probability $q_{i}$ is estimated as follows:

$$
q_{i} \triangleq \frac{\Theta\left(L\left(S_{i}^{\text {self }}\left(\xi_{i}\right), W_{\text {std }}\right), L\left(S(\xi), W_{\text {std }}\right)\right)}{\sum_{j=1}^{s n} \Theta\left(L\left(S_{j}^{\text {self }}\left(\xi_{j}\right), W_{\text {std }}\right), L\left(S(\xi), W_{\text {std }}\right)\right)} .
$$

\subsubsection{Generation-based character layout facsimileing}

If the set $\mathbf{L}_{\text {self }}$ is empty, it means we would have to generate $L(S(\xi), X)$ according to the spatial layout produced by other writers, namely those templates in $\mathbf{L}_{\text {others }}$. For each layout $L\left(S_{i}^{\text {other }}\left(\xi_{i}\right), W_{i}\right) \in \mathbf{L}_{\text {others }}$, we find its corresponding layout $L\left(S_{i}^{\text {other }}\left(\xi_{i}\right), W_{\text {std }}\right)$, i.e., the layout of the character $\xi_{i}$ written in the standard font (the "Kai" GB2312 style). We use (4) to derive their corresponding feature matrices, denoted as $\mathbf{F}_{x}^{i}=\mathbf{F}_{x}\left(L\left(S_{i}^{\text {other }}\left(\xi_{i}\right), W_{i}\right)\right)$ and $\mathbf{F}_{x}^{\text {std }}=$ 
$\mathbf{F}_{x}\left(L\left(S_{i}^{\text {other }}\left(\xi_{i}\right), W_{\text {std }}\right)\right)$ respectively. Based on these two feature matrices, we derive their difference matrix as $\mathbf{D i s}_{x}^{i} \triangleq$ $\mathbf{F}_{x}^{i}-\mathbf{F}_{x}^{\text {std }}$. And then we will be able to synthesize an overall feature difference for facsimileing the spatial layout of $L(S(\xi), X)$ based on the layout examples in $\mathbf{L}_{\mathrm{others}}$ as:

$$
\mathbf{D i s}_{x}^{\text {overall }} \triangleq \frac{\sum_{i=1}^{o n} \mathbf{D i s}_{x}^{i} \Theta\left(L\left(S_{i}^{\text {other }}\left(\xi_{i}\right), W_{\mathrm{std}}\right), L\left(S(\xi), W_{\mathrm{std}}\right)\right)}{\sum_{i=1}^{o n} \Theta\left(L\left(S_{i}^{\text {other }}\left(\xi_{i}\right), W_{\mathrm{std}}\right), L\left(S(\xi), W_{\mathrm{std}}\right)\right)} .
$$

Basically, Dis ${ }_{x}^{\text {overall }}$ is derived through weighted summing over $\operatorname{Dis}_{x}^{i}$ using $\Theta\left(L\left(S_{i}^{\text {other }}\left(\xi_{i}\right), W_{\text {std }}\right), L\left(S(\xi), W_{\text {std }}\right)\right)$ as the respective weight. Given Dis $_{X}^{\text {overall }}$ we can derive the feature matrix $\mathbf{F}_{x}$ on $L(S(\xi), X)$ as: $\mathbf{F}_{x}(L(S(\xi), X)) \triangleq$ Dis $_{x}^{\text {overall }}+$ $\mathbf{F}_{x}^{\text {std }}$. Through a similar way, we can derive $\mathbf{F}_{y}(L(S(\xi), X))$. Given these two feature matrices describing the layout of the bounding boxes of the constituent character elements of the character $\xi$ written in the writer $X$ 's personal handwriting style following the constructive approach of $S$, we can reversely compute the optimal sizes and locations of these bounding boxes which would produce these two feature matrices. To do this, we employ a gradient-descent-based optimization process with the target function to be minimized defined as the $L_{2}$ norm of the distance between the expected feature matrices as estimated above and the feature matrices of the layout relationship under optimization. The layout so found is our facsimile result through the generation-based approach.

\subsubsection{Hybrid method for spatial layout facsimileing}

If $\mathbf{L}_{\text {self }}$ is empty but $\mathbf{L}_{\text {others }}$ is not, we can only facsimile a spatial layout through the generation-based approach; if $\mathbf{L}_{\text {others }}$ is empty but $\mathbf{L}_{\text {self }}$ is not, we can only get one facsimiled spatial layout from the reuse-based approach. However, typically both approaches can produce a facsimile result, in which case we use a random selection process to choose one result. That is, we select the result produced by the reuse-based approach with probability $z$ and select the result produced by the generation-based approach with probability $1-z$. Here $z$ is calculated according to the similarity scores of the two sets $\mathbf{L}_{\text {self }}$ and $\mathbf{L}_{\text {others. }}$. More concretely, $z$ is defined as $z \triangleq \frac{A}{A+B}$ where $A \triangleq \frac{1}{s n} \sum_{j=1}^{s n} \Theta\left(L\left(S_{j}^{\text {self }}\left(\xi_{j}\right), W_{\text {std }}\right), L\left(S(\xi), W_{\text {std }}\right)\right)$ and $B \triangleq$ $\frac{1}{\text { on }} \sum_{j=1}^{o n} \Theta\left(L\left(S_{j}^{\text {other }}\left(\xi_{j}\right), W_{\text {std }}\right), L\left(S(\xi), W_{\text {std }}\right)\right)$.

\section{Optimal Selection Over Candidate Results}

It is obvious that the more parts of a character previously written by a writer that are available, the easier and more reliably the character can be facsimiled. This leads to a simple but effective heuristic: we define a facsimile reliability function $\Phi\left(S_{i}(\xi), X\right)$ for evaluating the reliability of facsimileing $\xi$ in $X$ 's personal handwriting style following $\xi$ 's $i$-th construction approach $S_{i}(\xi)=\widehat{\xi_{1}} \ldots \widehat{\xi_{n}} . \Phi\left(S_{i}(\xi), X\right)$ is then intuitively defined according to the percentage in the facsimile result that the user $X$ has previously written:

$$
\Phi\left(S_{i}(\xi), X\right) \triangleq \sum_{j=1}^{n} \frac{\phi\left(\widehat{\xi}_{j}, X\right) \operatorname{Area}\left(\widehat{\xi}_{j}\right)}{\operatorname{Area}\left(\hat{\xi}_{j}\right)}
$$

where $\operatorname{Area}\left(\widehat{\xi_{j}}\right)$ denotes the area occupied by $\xi$ 's character element $\widehat{\xi}_{j}$ and

$\phi\left(\widehat{\xi}_{j}, X\right) \triangleq\left\{\begin{array}{lll}1 & \text { if } X \text { has previously written } \widehat{\xi_{j}} ; \\ \max \left\{\lambda_{1}^{\mathrm{opt}}\left(\widehat{\xi}_{j}\right), \cdots, \lambda_{n w}^{\mathrm{opt}}\left(\widehat{\xi}_{j}\right)\right\} & \text { otherwise. }\end{array}\right.$

in which $\lambda_{i}^{\text {opt }}\left(\widehat{\xi_{j}}\right)$ 's are the optimal assignment over $\lambda_{i}$ 's as determined in Sec. 3.2 for facsimileing the character element $\widehat{\xi_{j}}$ following a generation-based approach. The rationale behind assigning $\max \left\{\lambda_{1}^{\text {opt }}\left(\widehat{\xi_{j}}\right), \cdots, \lambda_{n w}^{\text {opt }}\left(\widehat{\xi_{j}}\right)\right\}$ to $\phi\left(\widehat{\xi_{j}}, X\right)$ when the user has not previously written the character element is because the higher the $\max \left\{\lambda_{1}^{\mathrm{opt}}\left(\widehat{\xi}_{j}\right), \cdots, \lambda_{n w}^{\mathrm{opt}}\left(\widehat{\xi}_{j}\right)\right\}$, the more confident the algorithm is in facsimileing the character element through the generation-based approach via (3). This can be intuitively understood as the higher $\max \left\{\lambda_{1}^{\mathrm{opt}}\left(\widehat{\xi}_{j}\right), \cdots, \lambda_{n w}^{\mathrm{opt}}\left(\widehat{\xi}_{j}\right)\right\}$, the more likely $X$ 's writing style is similar to one of the reference writers $W_{i}$, in which case the generation-based facsimile approach behaves more like the reuse-based facsimile approach. Given the above facsimile reliability function, our algorithm selects the candidate facsimile result with the highest reliability score and outputs it as the final facsimile result.

\section{Experiment Results}

We first compare our calligraphy facsimile results with the original handwritings of two Chinese calligraphists in Figure 4 . In this experiment, only 24 other characters written by the respective calligraphists are accessible to our algorithm for learning. Our facsimile results are not exactly identical to the ones written by the calligraphists, which is to be expected. Nevertheless, for most of the characters, the facsimile results appear to be very close in appearance and style to the authentic handwritten ones. And then we present Figure 5 for examining the faithfulness of the facsimiled results produced by our algorithm when the algorithm has access to different sizes of training set of sample calligraphic characters. The analysis clearly shows the enhancement of our algorithm's performance when it has access to more characters written by the target calligraphist. The analysis results also suggest when our algorithm has seen 40 characters written by a writer, it can facsimile the handwriting of the writer to the degree such that the handwritten samples and the facsimiles are almost indistinguishable. Practically, it is not hard to collect 40 characters of handwriting by a person.

We also present the results of a facsimile experiment over a famous Chinese poem in Figure 6. As can be easily seen, a high degree of faithfulness has been achieved. Using the 


\section{两辰中秋欢饮达旦 两唇中秋欢饮达旦 丙辰中秋欢饮达旦 丙辰中秋欢饮达旦}

Figure 4: Facsimileing results compared with the original handwritings of two Chinese calligraphists. The first and third rows are the original handwritings of the two Chinese calligraphists; the second and fourth rows are the facsimiled results.

results of this experiment, we conducted a small-scale quantitative user study in the form of a quasi Turing test. We invited eight educated Chinese individuals to tell apart the facsimiled results by our algorithm from the authentic writings by the calligraphist. Six of them are computer science graduate students, and two of them are middle school literacy teachers. We showed them 16 sample characters written by the calligraphist, followed by 20 characters facsimiled by our algorithm. After that we showed them a set of 32 new characters and asked them to tell which ones are written by the calligraphist and which ones are facsimiles. Among these 32 characters, 17 characters are from authentic handwritings and 15 characters are machine facsimiled ones, as labeled $*$ in Figure 6 . Figure 7 shows the respective accuracies achieved by these eight individuals. Their accuracy rates are $62.5 \%, 53.1 \%, 46.9 \%, 68.8 \%, 43.8 \%, 34.3 \%$, $59.3 \%, 50.0 \%$ respectively, and the overall accuracy rate is $52.3 \%$. From these data we see that the best human judge achieves the accuracy of $68.8 \%$ while the poorest achieves about $34.3 \%$. The overall accuracy of these human judges is $52.3 \%$, which does not differ much from the accuracy of random guess. This shows the facsimile results are sufficiently indistinguishable from the authentic handwritten ones to people in our user study group.

Due to space limitation, more experiment results are available in our project website at http://www.cs.hku.hk/ songhua/facsimile/.

\section{Conclusion}

This paper presents our attempt to generate facsimiles of Chinese calligraphic writings through an example-based learning approach. The resultant intelligent graphical design system can facsimile calligraphic writings with a high degree of realism in most situations, as verified by the experiment results. For the future, we will continue to refine our algorithm, aiming at an even higher degree of facsimile

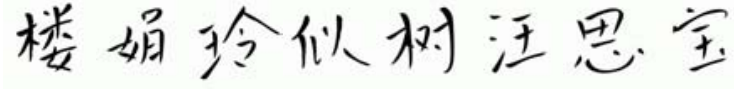

$\left(\begin{array}{llllllll}\left(A_{a}\right) & \left(B_{a}\right) & \left(C_{a}\right) & \left(D_{a}\right) & \left(E_{a}\right) & \left(F_{a}\right) & \left(G_{a}\right) & \left(H_{a}\right)\end{array}\right.$ 楼娟玲似树汪思宝

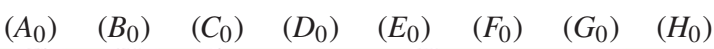
楼萌玲似树汪国至 $\left(\begin{array}{llllllll}\left.A_{10}\right) & \left(B_{10}\right) & \left(C_{10}\right) & \left(D_{10}\right) & \left(E_{10}\right) & \left(F_{10}\right) & \left(G_{10}\right) & \left(H_{10}\right)\end{array}\right.$ 楼娟玲似树汪, 思宝

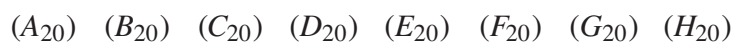
楼娟玲似树汪思宝

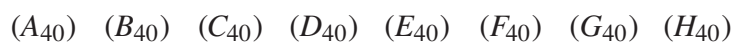

Figure 5: Analyzing the faithfulness of our algorithm's handwriting facsimile results using different sizes of training set. (A)-(H) show eight sets of such results. The rows from top to bottom respectively correspond to the authentic handwriting by a calligraphist $\left(X_{a}\right)$, facsimiled results when our algorithm has no access to any characters written by the calligraphist $\left(X_{0}\right)$, facsimiled results when our algorithm has learned 10, 20 and 40 characters written by the calligraphist as training samples $\left(X_{10}, X_{20}, X_{40}\right)$, respectively.

faithfulness. We also would like to extend our methods to cover more languages which might introduce new character features for our algorithm to consider. Right now, the styles of handwriting our algorithm is capable of facsimileing exclude the very cursive ones. The bottleneck is with the character decomposition and parameterization step. We plan to look for more advanced algorithmic approaches to attack the problem. Our generation process so far has not touched upon style consistency between adjacent characters, which is invariably an important consideration by human calligraphists.

\section{References}

[FTP03] Freeman W. T., Tenenbaum J. B., Pasztor E. C.: Learning style translation for the lines of a drawing. ACM Transactions on Graphics 22, 1 (2003), 33-46.

[GKK*97] Gader P. D., Keller J. M., KrishnapURam R., Chiang J.-H., Mohamed M. A.: Neural and fuzzy methods in handwriting recognition. Computer 30 , 2 (1997), 79-86.

[MAI93] M. O., ADACHI Y., IsHII N.: Fuzzy theory in hand writing learning system. In Proc. of International Joint Conference on Neural Networks (1993), pp. 766769 . 
明月几时有把㴗问青天不知

$(1 *)(2) \quad(3) \quad(4)\left(5^{*}\right)(6 *)(7 *)(8)(9 *)(10)(11)(12)$ 天上宫阙今夕是何年我欲乘

(13) (14) (15) $(16 *)(17)(18)(19 *)(20 *)(21)(22)(23 *)(24 *)$ 风归去又恐涼楼玉宇高处不

(25) (26) (27) (28)(29*)(30*)(31) (32) (33)(34*)(35) (36) 胜寒起舞弄清影何似在人间 $(37 *)(38)(39)(40 *)(41)(42)(43 *)(44)(45)(46)(47 *)(48)$ 转朱阁抵绮户照无眠不应有 $(49)(50 *)(51 *)(52 *)(53 *)(54)(55 *)(56)(57)(58)(59)(60)$ 恨何事偏向别时圆人有悲欢 (61) (62) (63) (64) (65)(66*)(67*)(68) (69) (70)(71*)(72) 离合月有阴晴圆缺此事古难 $(73 *)(74 *)(75)(76)(77)(78 *)(79)(80)(81)(82)(83)(84)$ 全但愿人长久千里共婵娟

$(85 *)(86 *)(87)(88)(89)(90)(91)(92 *)(93)(94)(95 *)$

Figure 6: A facsimile experiment over a famous Chinese poem by the great Chinese poet Shu Shi (1037AD-1101AD). The first four rows, i.e., (1)-(48), are written by a modern calligraphist while the remaining rows, i.e., (49)-(95), are facsimile results by our algorithm. In this experiment, our algorithm only has access to the characters (1)-(48) as training examples. Characters with * in their labels are used in the user study reported in Figure 7.

[MHN*99] Mano J., He L., NAKamura T., Enowaki H., Мutoh A., Iтон H.: A method to generate writingbrush-style Japanese Hiragana character calligraphy. In Proc. of IEEE International Conference on Multimedia Computing and Systems (1999), vol. 1, pp. 787-791.

[OSN05] OKabe Y., Saito S., NaKajima M.: Paintbrush rendering of lines using hmms. In GRAPHITE '05: Proc. International Conference on Computer Graphics and Interactive Techniques in Australasia and South East Asia (2005), pp. 91-98.

[WL01] WANG S.-Z., LEE H.-J.: Dual-binarization and anisotropic diffusion of Chinese characters in calligraphy documents. In ICDAR '01: Proc. of the 6th International Conference on Document Analysis and Recognition (2001), pp. 271-275.

[WLI05] Wong S. T. S., LeUnG H., IP H. H. S.: Modelbased analysis of Chinese calligraphy images. In Proc. of the 9th International Conference on Information Visualisation (2005), pp. 221-226.



Figure 7: The human identification results based on our facsimile experiment reported in Figure 6. Here we give the raw data recording each participant's performance. Each row in the bitmap corresponds to a user and each column corresponds to a character. Eight Chinese individuals participated in this user study, hence eight rows. Each user is asked to tell whether a character is written by the original writer or a machine facsimiled one, for a total of 32 characters. Hence the bitmap has 32 columns. The 32 characters used in the survey are marked by * in Figure 6. A black color in a block indicates the user made a correct guess, and white color for a wrong answer. The accuracy rates of the eight individuals are $62.5 \%, 53.1 \%, 46.9 \%, 68.8 \%, 43.8 \%, 34.3 \%$, $59.3 \%, 50.0 \%$ respectively, while the overall accuracy rate is $52.3 \%$. This shows the facsimile results are sufficiently indistinguishable from the authentic handwritten ones to people in our user study group.

[WWPF08] Wang Y., Wang H., Pan C., Fang L.: Style preserving Chinese character synthesis based on hierarchical representation of character. In Proc. of IEEE International Conference on Acoustics, Speech and Signal Processing (2008), pp. 1097-1100.

[XJLP07] XU S., Jiang H., LaU F. C.-M., PAN Y.: An intelligent system for Chinese calligraphy. In $A A A I$ '07: Proc. of the 22nd AAAI Conference on Artificial Intelligence (2007), pp. 1578-1583.

[XlCP04] Xu S., Lau F. C., Cheung K.-W., Pan Y.: Automatic generation of artistic Chinese calligraphy. In AAAI '04: Proc. of the 19th National Conference on Artificial Intelligence (2004), pp. 937-943.

[XlCP05] Xu S., LaU F. C. M., Cheung W. K., Pan Y.: Automatic generation of artistic Chinese calligraphy. IEEE Intelligent Systems 20, 3 (2005), 32-39.

[XLP03] XU S., LAU F., PAN Y.: Automatic artistic calligraphy generation. Technical Report, HKU-CSIS-TR2003-02, Department of Computer Science and Information Systems, The University of Hong Kong.

[YH96] YAMASAKI T., HatTORI T.: Computer calligraphy-brush written Kanji formation based on the brush-touch movement. In Proc. of IEEE International Conference on Systems, Man, and Cybernetics (1996), pp. 1736-1741.

[YP05] YU J., Peng Q.: Realistic synthesis of Cao Shu of Chinese calligraphy. Computers and Graphics 29, 1 (2005), 145-153. 